\title{
Geophysics
}

\section{Aurora seen in daylight}

\section{from Alan Johnstone}

THE long-standing question of whether aurora occurs during the day as well as the night has just been answered in a most convincing way. The answer has actually been clear from indirect evidence for many years, because the electrons, which cause the aurora when they cascade into the upper atmosphere, produce many other detectable effects as they excite atmospheric molecules to emit their characteristic light. In fact, aurora occurs all round the magnetic pole in an oval offset towards the nightside. It is there during the summer days of midnight sun as well as during the long weeks of darkness in winter. Sunlight seems to have no influence on the occurrence of aurora. The difficulty until now has been that none of the methods used to detect aurora gives a picture of its structure.

The aurora cannot be seen in daylight because the brightness of scattered light (the blueness of the sky) overwhelms the much fainter light from the aurora. At ultraviolet wavelengths (below $200 \mathrm{~nm}$ ), however, the background of scattered light is reduced both because the incoming solar radiation is weaker and because the radiation is totally absorbed by the atmosphere before penetrating it or being reflected from it.

Molecules excited by the impact of auroral electrons emit light at ultraviolet wavelengths, notably $\mathrm{N}_{2}$ in molecular bands between 132.5 and $150 \mathrm{~nm}$, and atomic oxygen with transitions at 130.4 and $135.6 \mathrm{~nm}$. These emissions cannot be seen from the ground because they too are absorbed by the atmosphere. They can, however, be seen from above by instruments carried on satellites because they occur at such a height that the light can escape upwards without much attenuation.

The first auroral images in ultraviolet light, obtained in full sunlight by the American polar-orbiting spacecraft Hilat, have just been published (Meng \& Huffman Geophys. Res. Lett. 4, 135; 1984). As the instrument looks down from a height of $820 \mathrm{~km}$ it scans a strip $5,000 \mathrm{~km}$ long stretching from horizon to horizon across the satellite track. The forward movement of the satellite builds up a picture from a series of parallel lines just as a television tube does. The intensity in a band only $3 \mathrm{~nm}$ wide is measured by a spectrometer which covers the range of wavelengths from 110 to $190 \mathrm{~nm}$. This narrow bandwidth improves the signal-tobackground ratio when the spectrometer is set on the most prominent features in the

Alan Johnson is in University College London Mullard Space Science Laboratory, Holmbury St Mary, Dorking, Surrey RH5 6NT. auroral spectrum. The proof of the technique is in the picture below which shows a typical auroral structure, familiar from the nightside images.
Now that the restrictions on auroral images imposed by sunlight have been removed, the exciting possibility emerges of obtaining pictures of the aurora simultaneously at both ends of a geomagnetic field line. This would require recording by spectrometers on at least two satellites. A survey of the extent to which the aurora is conjugate would provide a great deal of information about the processes responsible.

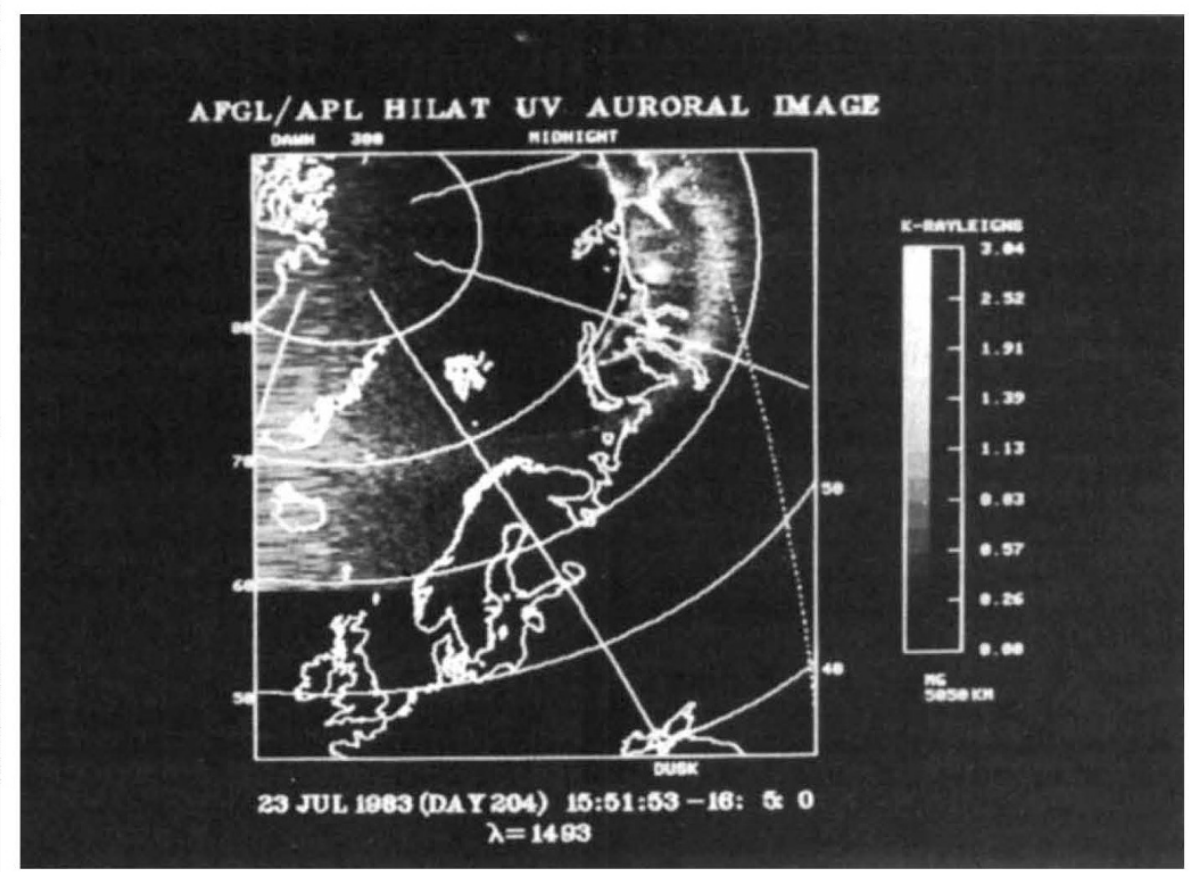

Hilat auroral imagery over sunlit summer polar region. The nightside, under full sunlight in summer, of the auroral oval is over the arctic coast of the Soviet Union. The day-night terminator on the Earth's surface is indicated by the dotted line. The picture is in geomagnetic coordinate. The land mass above $80^{\circ}$ geographic latitude is deleted in the picture.

\section{Plant science}

\section{Molecular studies forge ahead}

\section{from John Ingle}

EIGHT years and four meetings have passed since the first NATO/FEBS Advanced Study Institute provided a focus for the then fledgling science of plant molecular biology. The progress can honestly be described as dramatic. Research has progressed from the initial problems of DNA isolation and cloning, through nucleotide sequence analysis and vector construction to the use of site-directed mutagenesis for detailed study of regulatory and structural gene sequences. Physiological problems are now routinely addressed via the modulation of gene expression at both RNA and protein levels, and the transfer and efficient expression of tissue-regulated genes is an established fact. The impact and potential of molecular manipulations on the improvement of plants has attracted commercial interest in agricultural opportunities, a point amply illustrated by the strength and vigour of biotechnology com- pany participation at a recent meeting*.

The initial focus of plant molecular biology on the chloroplast has largely continued, producing a detailed analytical description of its genome. Functional characterization of the genome has been less successful, depending largely on the use of heterologous in vitro systems, such as Escherichia coli. The development of an homologous transcription system and characterization of the RNA polymerase activities responsible for expression of chloroplast genes, together with the identification of chloroplast promoter elements, represents a major advance (W. Gruissem, University of California, Berkeley), but a chloroplast transformation system is still urgently needed.

For nuclear genes a welcome main trend

"'Molecular form and function of the plant genome', NATO Advanced Studies Institute FEBS Advanced Course, 4-14 July, Renesse, The Netherlands. 